\title{
Fullerenes in Medicine; Will it ever Occur?
}

\section{Chris Kepley*}

Associate Professor, Joint School of Nanoscience and Nanoengineering, University of North Carolina at Greensboro, North Carolina, 27401, USA

Fullerenes represent a group of compounds having unique properties that make them attractive candidates for use as a platform for developing new medical applications. The carbon cage (usually $\mathrm{C}_{60}$ and $\mathrm{C}_{70}$ ) of empty cage fullerenes (Figure 1-left/middle) are being developed as therapeutics for disease processes such as multiple sclerosis, neurodegeneration, HIV infection, cancer, radiation exposure, ischemia, allergic disease, infectious disease, and general inflammation. Metallo-fullerenes (Figure 1-right; that have metals enclosed within the carbon cage) are being developed into new biomarker homing, diagnostic contrast agents for MRI. Lastly, a new class of theranostics are being developed that combine cell targeting capabilities/imaging with a therapeutic payload. Their inherent properties combined with their ability to be derivatized with side chains results in almost limitless new chemical structures making them ideal platform molecules for new solutions to basic biological problems. However, one of the biggest obstacles that have kept this class of compounds from potentially improving human health and reducing health care costs is the concern about toxicity. This has been due, in part, to the lack of standard structural relationships that affect biological outcomes of Fullerene Derivatives (FD).

Of course, toxicity considerations are implicit when contemplating human use for novel compounds such as fullerenes. To this end there have been a number of studies examining the toxicity using a myriad of fullerene preparations. However, the results of most of these studies are conflicting, inconclusive, and the subject of much debate. One of the most "damaging" fullerene studies (that was subsequently proven to be unfounded) exposed juvenile bass to un-derivatized $\mathrm{C}_{60}$, which is insoluble in water [1]. Unfortunately the authors did not include a control that provided insights into whether the observed effects were simply due to large aggregated particles/THF contaminants or whether they were specific to the chemical nature of $\mathrm{C}_{60}$. Yet there was widespread publicity that concluded fullerenes as a class could be toxic [2]. A more recent publication by the same group (without publicity) demonstrated that the originally observed "toxicity" was due to impurities in the sample [3]. Even more recently these original studies were "formally" debunked by a follow-up publication which stated that the original Oberdorster studies where "compromised by experimental artifacts" [4]. On the opposite end of the spectrum, studies in mice demonstrate that similar $\mathrm{C}_{60}$ preparations significantly increase the lifespan of mice [5]. Thus, it is difficult for researchers, and the general public, to determine if fullerenes are dangerous nanostructures that should be banned or a potential novel platform for developing new fountain-of-youth medicines. How can a class of compounds be simultaneously toxic and lifespan extenders?
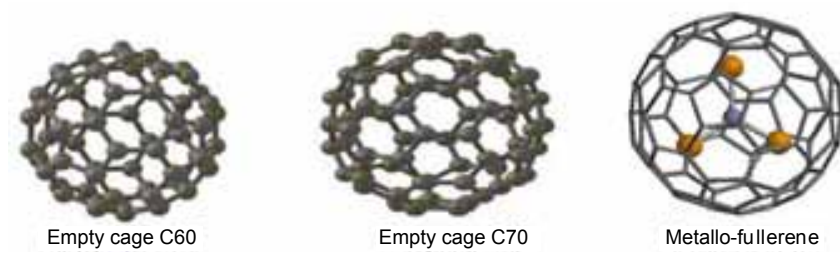

Figure 1: Representative fullerene structures.
Contributing to the confusion is that few studies examining toxicity use well characterized and highly purified material that are not mixtures of many isomers, aggregate sizes, and impurities. Most studies in which fullerenes are deemed to be toxic use starting material with little or no characterization (DLS, zeta potential, FTIR, etc.). In addition, no studies have compared differences in cage sizes $\left(\mathrm{C}_{60}\right.$ vs. $\mathrm{C}_{70}$ vs. $\mathrm{C}_{80}$ ); the latter two are much more likely to become US Food and Drug Administration (FDA) approved products due to their size being conducive to fewer numbers of isomers when adding side chain moieties. The FDA requires that every new chemical entity (NCE) must be evaluated separately; extrapolating toxicity (or non-toxicity) by categorizing compound mixtures and making generalizations about classes of compounds (as is the case with fullerenes) with many different isomers is not acceptable to the FDA. Anyone with a high school-level understanding of basic chemistry is aware that even extremely similar molecules can have different biologic activities. There are many examples where two isomers that are very similar have completely different biological behavior. For example, the tragedy of the drug thalidomide, where one isoform was an effective sedative and the mirror image isoform was teratogenic resulting in fetal defects, changed the face of drug testing. This applies to the studies with fullerenes where even extremely small changes in the core fullerene structure (through the addition of side-chain moieties) can result in the FD having completely different biological properties. This has been demonstrated repeatedly by several laboratories which highlight the difficulty in interpreting data gathered from extrapolating findings between even very similar compounds.

Complicating the task of bringing FD to the marketplace is the fact that the FDA does not have specific guidelines for products containing nanoscale materials. A report issued by the FDA Nanotechnology Task Force (July 2007) recommends guidance by various centers within the FDA for industries working with nanomaterials. Unlike "standard" drug products it is increasingly evident that at least in the area of characterization of nanomaterials used in drug products, different standards apply. Applying small molecule principles and methodologies to nanomaterials cannot be extrapolated in biological settings. The study stressed that biodistribution analysis should be at the core of any evaluation of products containing nanomaterials. These biodistribution studies, as recommended by the FDA, provide valuable information on where the nanoparticles are traveling and possibly accumulating, therefore subjecting those sites to increased likelihood of toxicological effects. It was also stressed in the 2007 report that most studies (using nanomaterials) are limited in that they are short-term,

*Corresponding author: Chris Kepley, PhD, Associate Professor, Joint School of Nanoscience and Nanoengineering, University of North Carolina at Greensboro, North Carolina, 27401, USA, E-mail: clkepley@uncg.edu

Received July 09, 2012; Accepted July 09, 2012; Published July 11, 2012

Citation: Kepley C (2012) Fullerenes in Medicine; Will it ever Occur? J Nanomed Nanotechol 3:e111. doi:10.4172/2157-7439.1000e111

Copyright: (c) 2012 Kepley C. This is an open-access article distributed under the terms of the Creative Commons Attribution License, which permits unrestricted use, distribution, and reproduction in any medium, provided the original author and source are credited. 
and might leave long-term effects unevaluated, especially because the long-term toxicity and effects for most nanoscale materials remain unknown. Furthermore, appropriate endpoints for in vitro assays are seen to be difficult to determine, as single cell types are often not sufficient for evaluation on the function or health of organs or tissues that are made up of multiple cell types, and given that numerous types of tissues are exposed to in the body. The major recommendation was that nanoscale material be characterized with respect to size (surface area and size distribution), chemical composition (such as purity and crystallinity), surface structure (surface reactivity, surface groups, coatings, etc.), solubility, shape, and aggregation. The protocols developed at the National Cancer Institute's, Nanotechnology Characterization Laboratories was recommended as being very useful in helping to characterize nanoscale materials and to develop standards and standardized methods for measuring nanoscale materials.

The potential for using fullerene-based medicines is substantial but concerns of toxicity have slowed the initial enthusiasm that surrounded their discovery. Only those studies using well characterized, single species "lead candidate" fullerene formulations can provide meaningful information regarding potential toxicological effects. Such studies are needed as the state of research today with fullerenes is shaped by studies such as these that address the observation "that extrapolation across similar nanoparticles will be dependent upon surface chemistry and concentration which may affect the degree of agglomeration and thus biological effects" [6]. Thus, more thorough studies will serve as a building block in developing a database that links surface functionalization chemistry of fullerene compounds to biologic function.

\section{References}

1. Oberdorster E (2004) Manufactured nanomaterials (fullerenes, C60) induce oxidative stress in the brain of juvenile largemouth bass. Environ Health Perspect 112: 1058-1062.

2. Barnaby JF (2004) New York Times.

3. Zhu S, Oberdorster E, Haasch ML (2006) Toxicity of an engineered nanoparticle (fullerene, C60) in two aquatic species, Daphnia and fathead minnow. Mar Environ Res 62: S5-S9.

4. Henry TB, Petersen EJ, Compton RN (2011) Aqueous fullerene aggregates (nC60) generate minimal reactive oxygen species and are of low toxicity in fish: a revision of previous reports. Curr Opin Biotechnol 22: 533-537.

5. Quick KL, Ali SS, Arch R, Xiong C, Wozniak D, et al. (2008) A carboxyfullerene SOD mimetic improves cognition and extends the lifespan of mice. Neurobiol Aging 29: 117-128.

6. Saathoff JG, Inman AO, Xia XR, Riviere JE, Monteiro-Riviere NA (2011) In vitro toxicity assessment of three hydroxylated fullerenes in human skin cells. Toxicol In Vitro 25: 2105-2112. 